Canadian Journal of Plant Science Revue canadienne de phytotechnie

The molecular basis for an ancient colour mutant in sweet
pea (Lathyrus odoratus)

\begin{tabular}{|r|l|}
\hline Journal: & Canadian Journal of Plant Science \\
\hline Manuscript ID & CJPS-2017-0238.R1 \\
\hline Manuscript Type: & Article \\
\hline Date Submitted by the Author: & 06-Oct-2017 \\
\hline Complete List of Authors: & $\begin{array}{l}\text { Xue, Xinxin; University of British Columbia, Department of Botany } \\
\text { Cronk, Quentin; University of British Columbia, Department of Botany }\end{array}$ \\
\hline Keywords: & Lathyrus odoratus, sweet pea, anthocyanin, delphinidin, floral mutation \\
\hline & \\
\hline
\end{tabular}

SCHOLARONE ${ }^{m}$

Manuscripts 


\section{The molecular basis for an ancient colour mutant in sweet pea}

\section{(Lathyrus odoratus)}

Xinxin Xue, and Quentin C.B. Cronk

Xinxin Xue and Quentin Cronk. Department of Botany, The University of British Columbia, 6270 University Blvd., Vancouver, BC V6T 1Z4, Canada.

Corresponding author: Quentin Cronk (email: quentin.cronk@ubc.ca).

Address: Department of Botany, The University of British Columbia, 6270 University Blvd., Vancouver, BC V6T 1Z4, Canada

Telephone: 1-604-781-6740 


\begin{abstract}
The classic A1 locus in sweet pea (Lathyrus odoratus) was investigated by Bateson, Punnett and Saunders in the early $20^{\text {th }}$ century history of Mendelian genetics. The mutation, in the form of the pink and white cultivar 'Painted Lady', is known from the $18^{\text {th }}$ century. We show that this locus is associated with a single base pair mutation (332 G/A) in the flavonoid 3',5'hydroxylase $\left(F 3^{\prime} 5^{\prime} H\right)$ gene. This results in an amino acid change (111 glycine/aspartic acid) in the conserved substrate recognition site 1 (SRS1) of the enzyme. The mutant flower lacks the blue pigment delphinidin and is thus pink and white, rather than purple and blue as in the wild type. This single amino acid change at a functionally important site appears to convert the enzyme from primary $\mathrm{F}^{\prime} 5^{\prime} \mathrm{H}$ activity to a relatively efficient $\mathrm{F}^{\prime} \mathrm{H}$, as suggested by heterologous transformation into Arabidopsis PAPID (a mutant line that produces anthocyanin constitutively).
\end{abstract}

Key words: floral mutation, delphinidin, anthocyanin, Lathyrus odoratus 


\section{Introduction}

\section{The sweet pea}

The sweet pea (Lathyrus odoratus) is an important floriculture crop of temperate regions. At the beginning of the 20th century, pioneer genetic studies using sweet pea revealed at least 18 hereditary factors, the study of which (specifically flower colour) led to the discovery of genetic linkage (Punnett, 1923, Bateson et al., 1906), although the results were not immediately interpreted correctly. Sweet peas are self-compatible and easy to grow, with cultivars representing inbred lines, which made them attractive to early geneticists.

The sweet pea has zygomorphic flowers typical of papilionoid legumes (Fabaceae), with a corolla differentiated into a large dorsal petal (standard, or banner), a pair of lateral petals (the wings) and a fused pair of ventral petals (the keel). The wild type cultivar 'Cupani' ('CP') is named after the Sicilian naturalist Francesco Cupani (1657-1710), who around 1695 sent the British horticulturist Robert Uvedale (1642-1722) a batch of sweet pea seeds, thus introducing the plant to the gardens of northern Europe. The flower of wild type L. odoratus is bicolourous with a reddish-purple standard and blue wings (Figure 1) resulting from their anthocyanin composition (Lawrence et al., 1941, Beale et al., 1939). The anthocyanins of the sweet pea are located in the vacuole of the petal epidermis (Figure 1). A mutant with pink standard and white wings, traditionally called 'Painted Lady' ('PL') first appeared around 1730 (Miller, 1754), and was illustrated in the $19^{\text {th }}$ century (Redouté, 1833). This phenotype was found to be controlled by a single locus (Bateson et al., 1906), named A1 (Punnett, 1923), which is tightly linked to a floral 
shape gene 'A3', now known to be the floral developmental gene CYCLOIDEA (Woollacott, 2010).

\section{Flower colour}

In angiosperms, flower colour display relies on the pigment production of flavonoids (most of the visible spectrum), carotenoids (yellow to red) or betalains (yellow or red), together with factors such as vacuole $\mathrm{pH}$, copigments and metal ions. The phenolic flavonoid compounds are secondary metabolites which are present in both vegetative and reproductive tissues of the plants. The flavonoid pathway is highly conserved in plants and has been studied extensively at both biochemical and molecular levels with most of the pathway genes cloned (Tanaka et al., 2009, Winkel-Shirley, 2001). Different subclasses of the flavonoids confer a wide spectrum of colour: anthocyanins (orange, red, magenta, violet and blue), aurones and chalcones (yellow), flavones and flavonols (colourless or very pale yellow).

Figure 2 shows a partial scheme of the flavonoid pathway. The six major anthocyanidins are classified into three groups based on the increasing number of hydroxyl groups on the B ring: (1) pelargonidin, (2) cyanidin and peonidin, and (3) delphinidin, petunidin and malvidin. As the number of B-ring hydroxyl group increases, the flower becomes bluer due to a bathochromic shift whereby the maximal absorption spectrum shifts towards longer wavelength (WinkelShirley, 2001). Delphinidin is the main blue pigment. However, it should be noted that blue can also result from co-pigmentation or $\mathrm{pH}$, or the two combined. Through co-pigmentation with metal ions, the pink cyanin (glycoside of cyanidin) can form blue protocyanin, as in Centaurea cyanus (Takeda et al., 2005) and a rise in $\mathrm{pH}$ can result in most anthocyanins appearing blue. The pathway flux to delphinidin production is directed by flavonoid 3',5'-hydroxylase (F3'5'H, CYP75B, EC 1.14.13.88). Mutations that affect expression of the F3'5'H gene are often 
associated with cyanidin-based colour shifts. F3 $^{\prime} 5^{\prime} \mathrm{H}$, along with flavonoid $3^{\prime}$-hydroxylase (F3'H, CYP75A, EC 1.14.13.21) and flavone synthase II (FNSII, CYP93B), belongs to the cytochrome P450 (CYP450)-dependent monooxygenase family, a large group of membrane-bound enzymes common to plants and animals. Evolutionarily, $\mathrm{F}^{\prime} 5^{\prime} \mathrm{H}$ extends the function of $\mathrm{F}^{\prime} \mathrm{H}$ providing additional 5' hydroxylation on the B ring of anthocyanin or flavonol precursors. The C-terminal end of $\mathrm{F}^{\prime} \mathrm{H}$ and $\mathrm{F3}^{\prime} 5^{\prime} \mathrm{H}$ distinguishes their functions with substitution of one or two amino acids changing the ratios of $3^{\prime}, 5^{\prime}$ - to $3^{\prime}$-hydroxylation (Seitz et al., 2007). Most species possess a single copy (or at most two copies) of $\mathrm{F}^{\prime} 5^{\prime} \mathrm{H}$. An exception is grape (Vitis vinifera), which has as many as 16 copies and this may have contributed to the strong accumulation of anthocyanins in grape skin (Falginella et al., 2010). Rat'kin and Tarasov (2010) reported two copies of F3'5'H (E and E1) in sweet peas, with only one being functional.

In plants generally, the evolutionary transition from blue to red flowers (as in the PL mutant of sweet pea) is asymmetrical with almost no reversal observed across studies (Wessinger and Rausher, 2015). However, reversion to blue may occur when the F3'5'H function is acquired through neofunctionalization of F3'H (Seitz et al., 2006).

The aim of this study is to identify the A1 locus of L. odoratus (that controls the PL phenotype) by, first, determining through anthocyanin profiling whether the lost blue pigment is delphinidin, and secondly, investigating whether changes in expression or sequence of the 'delphinidin gene' F3'5'H are associated with the pink mutant phenotype. For functional verification, we use in vivo transformation of the Lathyrus $\mathrm{F}^{\prime} 5^{\prime} \mathrm{H}$ into an Arabidopsis mutant line (PAP1D) that over-expresses anthocyanin biosynthetic genes. Arabidopsis has no endogenous $\mathrm{F}^{\prime} 5^{\prime} \mathrm{H}$. 


\section{Materials and Methods}

\section{Tissue sampling and RNA extraction}

Plants were grown from commercially sourced seed and maintained in horticultural greenhouse at the University of British Columbia (UBC) with ambient light and temperature. One wild type cultivar ' $\mathrm{CP}$ ' was crossed with a pink mutant 'PL' to yield six F1 hybrids that were allowed to self-pollinate in an insect-proof greenhouse to generate an F2 population. A total of $40 \mathrm{~F} 2$ plants were scored for colour phenotype and F3'5'H genotype.

\section{Pigment analysis}

Flowers were harvested just before or at anthesis (stages 5-6, Figure 3a) into silica gel desiccant. The standard and wing petals were analyzed separately. Each sample contained three flowers from one individual plant that were pooled together as one biological replicate. A total of three biological replicates were included for each cultivar. Water-soluble pigments were extracted with $1 \mathrm{ml}$ methanol: $\mathrm{H} 2 \mathrm{O}$ : acetic acid $(85: 15: 0.5, \mathrm{v} / \mathrm{v} / \mathrm{v})$ by soaking overnight at $4{ }^{\circ} \mathrm{C}$. The solution was centrifuged to retain the supernatant. The extract was acid hydrolysed with $2 \mathrm{M} \mathrm{HCl}$ for 30 mins in a waterbath $\left(80^{\circ} \mathrm{C}\right)$ to remove the sugar residues. Precipitates were centrifuged down and the supernatant was kept in the dark at $-20^{\circ} \mathrm{C}$ until processing.

HPLC-MS analysis was carried out using a ZORBAX SB-C18 reverse phase column (4.6 × $50 \mathrm{~mm}, 1.8 \mu \mathrm{m}$, Agilent). The crude extract was eluted at a rate of $1 \mathrm{ml} \mathrm{min}-1$ with $87 \%$ solvent A [H2O/FA (folic acid), 98:2, v/v] and 13\% solvent B (acetyl nitrate/FA, 98:2, v/v) for 4 min, followed by an elution gradient from $23 \% \mathrm{~B}$ for $2 \mathrm{~min}$ to $90 \% \mathrm{~B}$ for $1 \mathrm{~min}$, and then back to $13 \% \mathrm{~B}$. The eluate 
absorbance at 357-373 $\mathrm{nm}$ and 510-530 $\mathrm{nm}$ was determined using an Agilent 1100 Diode Array Detector (DAD). Technical quadruplicates with injection volume of $5 \mu 1$ were run for all samples. The elute absorbance at 357-373 $\mathrm{nm}$ (for flavonol aglycones) and 510-530 nm (for anthocyanidins) was determined using DAD. Accurate mass of the eluent was then determined by mass spectrometry (MS). Anthocyanidins and flavonol aglycones were examined at the positive and negative ionization modes respectively, following traditions. Peak identification was made by overlaying the MS chromatogram onto the LC chromatogram of a given sample, and pigment was assigned to each sample based on the retention time (TR) and mass to charge ratio $(\mathrm{m} / \mathrm{z})$ of the reference standards and online database (massbank.jp). The criteria for pigment assignment are listed in Table 1. The relative abundance of an identified pigment was determined by calculating the total area under its peak absorption

\section{Extraction of nucleic acid, cDNA synthesis and PCR}

For RNA extraction, fresh petals of $L$. odoratus were harvested at late bud stages S3-4 (Figure 3a) by flash freezing in liquid nitrogen. Standard and wing petals were analyzed separately. RNA was extracted with Pure Link Plant RNA Reagent (Invitrogen) following the manufacturer's protocol. Total RNA was treated with DNase twice using a Turbo DNA-free kit (Ambion). A firststrand cDNA was synthesized from total RNA (500 ng) using RevertAid First Strand cDNA Synthesis Kit (Fermentas) using supplied oligo(dT)18 primer and following the manufacturer's protocol. Genomic DNA from fresh leaf tissues was extracted using a modified CTAB protocol. A partial sequence of L. odoratus F3'5'H was obtained by RNA-seq (Illumina sequencing of total flower cDNA) with assembly into contigs using Trinity. The transcript sequences were then used to design primers. Sequences at the two ends of L. odoratus $\mathrm{F}^{\prime} 5^{\prime} \mathrm{H}$ were confirmed using the First 
Choice RLM-RACE kit (Ambion) following manufacturer's instructions. The full sequence is available on GenBank (accession number: \#\#\#\#\#\#\#).

The L. odoratus transcriptome library was obtained as follows: total RNA was isolated from flowers of several individuals at the mature bud stage. RNA was treated with DNase twice using a Turbo DNA-free kit (Ambion). The quality of combined RNA from two independent extractions was then determined using a 2100 Bioanalyzer (Agilent). The sample was submitted for wholetranscriptome sequencing by Cofactor Genomics (St Louis, MO) using the Illumina HiSeq platform.

Primers for RT-PCR were designed to span between two exons to eliminate false signals given by DNA contamination. F3'5'H forward primer F9: 5'-ACCAGGTCCAAAGGGTTATC, reverse primer R8: 5'-AATGTAAGCAGATGGAGACA. Each PCR amplification uses a reaction mixture containing $0.5 \mathrm{mM}$ of each primer, $10 \mu \mathrm{PCR}$ buffer (Fermentas), $2.0 \mathrm{mM} \mathrm{MgCl} 2,0.25 \mathrm{mM}$ of each dNTP, and 5 units of recombinant Taq polymerase (Fermentas). PCR was performed for 25 or 30 cycles with each cycle condition: 30 s at $94^{\circ} \mathrm{C}, 30$ s at $53^{\circ} \mathrm{C}, 1 \mathrm{~min}$ at $72^{\circ} \mathrm{C}$. $\mathrm{PCR}$ products were run on $1.5 \%$ agarose gels $(0.5 \mu \mathrm{TAE}$ buffer) with $3 \mu \mathrm{l}$ GelRed (Biotium) for band separation and visualization. PCR product was purified using a QIAquick Gel Extraction Kit following the manufacturer's instructions.

F2 co-segregation analysis

Selfed progenies of the six F1 plants were scored for colour and $\mathrm{F}^{\prime}{ }^{\prime} 5^{\prime} \mathrm{H}$ genotypes using the restriction enzyme HhaI (Fermentas), which digests the $332 \mathrm{G}$ in ' $\mathrm{CP}$ ', but not the mutant $332 \mathrm{~A}$ in 'PL'. Primers used for genotyping were F: 5'-ACCAGGTCCAAAGGGTTATC), R: 5'-

CCTTGAAGATCCAACCAAGC. The PCR products of two individuals were sequenced to confirm the genotype. 
Binary vector construction, transformation into Arabidopsis thaliana

Two expression constructs, 35S:cpF3'5' H (wild type) and 35S:plF3'5'H (mutant type) were generated using the binary vector pCAMBIA-1300. The full-length coding regions of F3'5' $\mathrm{H}$ were amplified from ' $\mathrm{CP}$ ' and 'PL' cDNA, using the forward primer 5'CCGGAATTCATGATACTCACCCTATACCAAACG and the reverse primer 5'GCTCTAGACTAAGGAACATAAGCAGATGGAG. The entire CDS of F3'5'H were subsequently inserted between the EcoRI and XbaI sites of pCAMBIA-1300. DNA inserts and pCAMBIA-1300 (3 $\mu 1$, courtesy of Yuti Cheng and Xin Li, UBC) were double digested with XbaI and EcoRI at $37{ }^{\circ} \mathrm{C}$ for $4 \mathrm{~h}$ (DNA inserts) or 12-16 h (vector) to ensure complete digestion. Digested products were recycled from a 1\% agarose gel using Gel Extraction Kit (Fermentas). Inserts with appropriate overhangs were then ligated into the binary vector pCAMBIA-1300 with sticky ends. The molar ratio of insert:vector was 3:1 (for $30 \mathrm{ng}$ of $7500 \mathrm{bp}$ vector, $18 \mathrm{ng}$ of $1500 \mathrm{bp}$ insert was added). Ligation was done at $16{ }^{\circ} \mathrm{C}$ overnight.

Agrobacterium-mediated gene transformation was carried out with floral dip method (Clough and Bent, 1998). Wild type Arabidopsis (col-0) was transformed once with 'CP'- and 'PL'-based 35S:F3'5'H constructs. Basta and Kanamycin resistant plants were selected over two generations for homozygous T2 plants. Two such T2 homozygotes from each cross were then crossed with PAP1-D (AT1G56650), a dominant homozygote mutant that over-expresses PAP1 (MYB75), a positive regulator of the genes involved in anthocyanin synthesis (Borevitz et al., 2000, Tohge et al., 2005). The cross yielded double heterozygous F1. A control line was generated crossing wild type with PAP1-D so that the control plants were heterozygous at PAP1-D locus as well. Leaves and roots were then collected from at least six mature F1 plants and control plants into silica gel for pigment analysis. RT-PCR was carried out to verify the expression of the sweet pea $\mathrm{F3}^{\prime} 5^{\prime} \mathrm{H}$ in the transgenics. Amplification of Arabidopsis ACTIN1 was used as control with forward primer: 5'CGATGAAGCTCAATCCAAACGA and reverse primer 5': CAGAGTCGAGCACAATACCG. 


\section{Results}

The pink mutant 'PL' lacks delphinidin, but produces more cyanidin

Just prior to and at anthesis (stage 5-6, Figure 3a), both cultivars showed an overall reciprocal change of anthocyanidin and flavonol contents in the two floral parts: anthocyanidins were much higher in the standard petal than in the wings and the reverse was true for the flavonols, contributing to the bicolourous pattern (Figure 1). Total anthocyanidin in 'CP' was 1.4 8.7 times that of 'PL', while the total flavonol ratio of ' $\mathrm{CP}$ ' to ' $\mathrm{PL}$ ' was $1.3 \sim 5.5$, indicating more pigment production in 'CP'. The main anthocyanidins in 'CP' were delphinidin and its derivatives in both standard and wings whereas 'PL' produces mainly the cyanidin series, predominately in the standard, with malvidin detected at a low level in the standard (Figure 3b). The shift from delphinidin to cyanidin indicates a reduced $\mathrm{F}^{\prime}{ }^{\prime}{ }^{\prime} \mathrm{H}$ activity in 'PL'. At the same time, the cyanidin content of 'PL' was 3.4$9.9 \times$ that of ' $\mathrm{CP}$ ' in the standard. This indicates the mutation, while abolishing delphinidin pathway, conversely boosts cyanidin production.

Quercetin was the major flavonol in both cultivars, followed by kaempferol. Only a trace amount of myricetin was detected in ' $\mathrm{CP}$ ' and it was not detected in 'PL'. Note that myricetin is a $\mathrm{B}$ ring tri-hydroxylated flavonol (Figure 2) whose production requires the action of F3'5' H.

A non-synonymous mutation in F3'5'H is associated with the pink colour in ' $P L$ '

As a lack of delphinidin may be due to a loss of function in $\mathrm{F}^{\prime} 5^{\prime} \mathrm{H}$ (Figure 2), this gene was investigated. L. odoratus F3'5'H CDS was determined to be 1548 bp long (513 aa) consisting of two exons (912 and $633 \mathrm{bp}$ in length). The predicted coding sequence of sweet pea $\mathrm{F}^{\prime} 5^{\prime} \mathrm{H}$ is $99 \%$ 
identical to that of Pisum sativum and Medicago truncatula and 79\% identical to Glycine max. Arabidopsis does not have an F3'5'H but it has an F3'H (CYP75B1) (which shares 48\% identity with the sweet pea F3'5'H) encoded by TRANSPARENT TESTA 7 (TT7) (Schoenbohm et al., 2000).

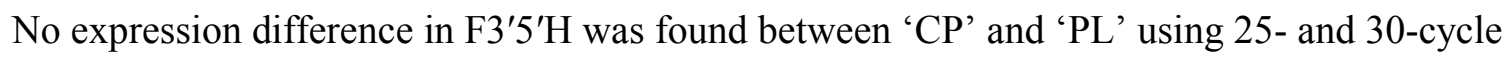
RT-PCR (Figure 4a). No difference was detected between different floral parts either. However, by sequencing, it was found that 'PL' carries a non-synonymous substitution (G/A) in the first exon 332 bp downstream of the ATG start site. This is predicted to change the amino acid from glycine to aspartic acid at codon 111, at a highly conserved site in the Substrate Recognition Site1 (SRS1, Figure 5b) (Seitz et al., 2006). F2 co-segregation analysis showed a simple Mendelian inheritance pattern for both the 'PL' phenotype and the non-synonymous mutation (332 G/A). In a total of 40 F2 plants, the 'PL' phenotype segregated in $100 \%$ of cases with the point mutation (Table $2, \mathrm{n}=11$ mutant plants, and Figure 4b). Therefore, the G/A mutation in $\mathrm{F}^{\prime}{ }^{\prime} 5^{\prime} \mathrm{H}$ is associated with the 'PL' phenotype, which indicates that it is either the causal mutation or a marker linked to the causal mutation.

Elevated production of cyanidin in the 'PL' F3'5'H-transformed Arabidopsis PAP1-D line

The Arabidopsis PAP1-D (Production of Anthocyanin Pigment 1-Dominant, MYB75) is a mutation affecting anthocyanin and other secondary metabolites synthesis (Shi and Xie, 2010). PAP1-D plants showed purple colour in all developmental stages. F1 control plants from a cross between PAP1-D and wild type Columbia showed the PAP1-D phenotype, confirming dominance of PAP1-D. Contrary to expectation, when the construct expressing 'CP'-F3'5'H under a 35S promoter was transformed into a heterozygous PAP1-D, the plants failed to produce the delphinidin series, but instead produced much higher cyanidin than the control (Figure 6). The 'PL'-F3'5'H-transformed line produced cyanidins in $>5 \times$ higher amounts than the control. And this cyanidin production in the 'PL'-F3'5'H transgenic line was also considerably higher than that in the 'CP'-F3'5'H-transformed 
line, showing that there is a functional difference between the PL and the CP enzymes. Quercetin and kaempferol accumulation was also considerably higher in the 'PL'-F3'5'H transgenic lines compared to either the control or the ' $\mathrm{CP}$ '-F3'5' $\mathrm{H}$ transgenics. All lines produced more kaempferol than quercetin, and no myricetin was detected.

\section{Discussion}

A non-synonymous mutation in ' $P L^{\prime}-F 3^{\prime} 5^{\prime} H$ is present at a structurally critical site

The pink colour in 'PL' is associated with a single-base substitution that has led to a change from small non-polar glycine to a polar bulky aspartic acid (G111D). F3'5'H is therefore highly likely a candidate for the A1 locus. The proposal that this is the causal mutation, rather than a marker closely linked to the causal mutation, is supported by further evidence. The same non-synonymous mutation $(\mathrm{G} / \mathrm{A})$ at the same position in the $\mathrm{F}^{\prime} 5^{\prime} \mathrm{H}$ exon (bp 332) resulting in a similar amino acid change (glycine to glutamic acid), has been associated with a pink Pisum sativum mutant generated by fast neutron bombardment (Moreau et al., 2012). This shows a remarkable parallelism at both phenotypic and molecular levels in two closely related species from the pea family. The point mutation in Lathyrus $\mathrm{F}^{\prime} 5^{\prime} \mathrm{H}$ does not affect transcription (Figure 4a), and the small amount of malvidin in 'PL' standard suggests it is a leaky, instead of a null, mutation of $\mathrm{F}^{\prime} 5^{\prime} \mathrm{H}$ which limits, but does not abolish, the original protein function.

Substrate recognition sites (SRS) in F3'5'H are highly conserved. SRS1, 2 and 4 (Nterminal) contact the distal aliphatic part, while SRS4 (C-terminal), 5 and 6 contact the aromatic ring of their respective substrates (Okinaka et al., 2003, Rupasinghe et al., 2003). In sweet pea 
cultivar 'PL', the substitution of a glycine (G111) by an aspartic acid in SRS1 is likely to affect substrate binding. Glycine is a unique amino acid as its side chain contains hydrogen instead of carbon, making it more flexible. Glycine can therefore occupy locations prohibited to other amino acids such as tight turns in structures. Indeed, by multi-species alignment (Figure $5 \mathrm{~b}$ ), this G111 corresponds to the Arabidopsis CYP75B (F3'H) glycine 105, in $\underline{\text { PNSG }}$ AKHㅆ of SRS1, a residue predicted to contact substrate dihydrokaempferol (DHK) (Rupasinghe et al., 2003). Mutation from glycine to the structurally distinct aspartic acid at this location may therefore alter substrate binding efficiency.

Overexpression of sweet pea wild type F3'5'H increases the accumulation of cyanidin and fails to produce delphinidin in Arabidopsis

The ' $\mathrm{CP}$ '-F3'5'H transgenic lines produced more cyanidin than the controls. This is unsurprising as it is well known that $\mathrm{F}^{\prime} 5^{\prime} \mathrm{H}$ may have $\mathrm{F}^{\prime} \mathrm{H}$ activity and that a few amino acid substitutions may convert one to the other (Seitz et al., 2007). The two genes are closely related having diverged from a common ancestor and reversals of function are known in nature (Seitz et al., 2006). Senecio cruentus F3'5'H for instance, when transformed into Chrysanthemum, fails to produce delphinidin but increases cyanidin production (He et al., 2013). What is more surprising is the complete lack of delphinidin production by wild type Lathyrus F3'5'H when transformed into Arabidopsis background.

For transformation, we used PAP1 over-expressing lines of Arabidopsis. PAP1 (MYB75) induces the expression of anthocyanin synthesis genes in all vegetative tissues across multiple developmental stages in Arabidopsis (Shi and Xie, 2010). However, even with this boost of substrate, the F3'5'H-transformed PAP1D lines failed to accumulate delphinidin, but did 
accumulate increased cyanidin. Because $\mathrm{F}^{\prime} 5^{\prime} \mathrm{H}$ was successfully expressed in the transgenic lines (Figure 6a), this could not be due to lack of expression. A lack of enzyme activity is also unlikely because the transformants show increased levels of cyanidin. With the enhanced $3^{\prime}$ hydroxylation in the $35 \mathrm{~S}:$ 'PL'-F3'5'H transgenic line, it is more likely that the failure to produce delphinidin happened at a functional control level.

The strong 3' hydroxylation coupled with a lack of 5' hydroxylation could have several possible causes, as listed below.

(1) The reaction of $\mathrm{F}^{\prime} 5^{\prime} \mathrm{H}$ could require a specific NADPH-CYP450 reductase that is absent in Arabidopsis. In animals, electron transfer to all the P450s is carried out by a single reductase (Benveniste et al., 1991), and for the 244 P450s in Arabidopsis (Bak et al., 2011), there are only two confirmed P450 reductases (ATR): ATR1 and ATR2-2 (Mizutani and Ohta, 1998, Urban et al., 1997, Varadarajan et al., 2010).

(2) There could be competition of endogenous $\mathrm{F}^{\prime}{ }^{\prime} \mathrm{H}$ with $\mathrm{F}^{\prime} 5^{\prime} \mathrm{H}$ for common substrates. Arabidopsis has a functional F3'H encoded by $\mathrm{tt} 7$ (Schoenbohm et al., 2000) which is active in the PAP1-D line which shows purple vegetative tissues. However, the strong apparent boosting of cyanidin production by exogenous $\mathrm{F}^{\prime} 5^{\prime} \mathrm{H}$ indicates that it competes successfully for substrates.

(3) Arabidopsis is not an appropriate host for sweet pea $\mathrm{F}^{\prime} 5^{\prime} \mathrm{H}$ for reasons other than the lack of a suitable reductase suggested above. Studies have shown that gene origin is important to the successful transformation of $\mathrm{F}^{\prime} 5^{\prime} \mathrm{H}$ with some host-donor pairs more successful in producing delphinidin-series than others (Okinaka et al., 2003, Katsumoto et al., 2007, He et al., 2013). One possibility is that substrate specificity for DFR is different between sweet pea and the Arabidopsis host. Although DFR in many species can use all three dihydroflavonols efficiently 
(Tanaka et al., 1995, Stich et al., 1992), sometimes however, it favors DHK over DHQ and DHM, e.g. in Antirrhinum and Gerbera, or vice versa such as in Petunia (Forkmann and Ruhnau, 1987) and Cymbidium (Johnson et al., 1999). The proposed mechanism is through a single residue at position 133, with an aspartic acid having a stronger affinity for DHQ and DHM while an asparagine favours DHK (Johnson et al., 2001). Arabidopsis DFR is coded by a single copy gene tt3 with asparagine (DHK inclined) at position 133. It is possible that this is suboptimal for delphinidin production.

One possibility for answering these questions may lie in further experiments using in vitro functional analysis of the enzymes or transformation into more natural hosts, i.e. Pisum or Lathyrus. Transformation of Pisum and Lathyrus has been reported (e.g. Švábová and Griga 2008; Barik, Mohapatra and Chand 2005), but is technically challenging, and was not attempted in the present study. For in vitro studies, heterologous expression of sweet pea $\mathrm{F}^{\prime} 5^{\prime} \mathrm{H}$ could be attempted in either yeast (Saccharomyces cerevisiae) or bacteria (Escherichia coli). As a eukaryote, yeast is advantageous in having similar processing and compartmentalization of proteins. However, due to codon bias (Hoekema et al. 1987) plant P450 proteins may be difficult to express unless their sequence is codon optimized (Plotkin and Kudla 2011; Wessinger and Rausher 2014). E. coli could also be used for expression of plant P450. In this case the predicted N-terminal transmembrane helix of the plant P450 can be modified to mimic the architecture of soluble bacterial P450s (Barnes et al. 1991).

Alteration of the SRS1 domain of sweet pea F3'5'H greatly increased the accumulation of cyanidin in a heterologous system 
The cyanidin content in the mutant 'PL'-F3'5'H transgenic lines was not only 5-6× that of the control, it was also considerably higher than the wildtype 'CP'-F'3'5' H transgenic lines. All F3'5'H enzymes have some F3'H activity (Seitz et al., 2006), but it is remarkable that the alteration of a single amino acid in the $\mathrm{F}^{\prime} 5^{\prime} \mathrm{H}$ apparently increases the propensity of the enzyme for 3' hydroxylation, and greatly increases cyanidin production over wild-type, indicating that this mutation is biochemically significant. The change from glycine to aspartic acid at residue 111 is apparently more a 'gain-of-function' mutation to make more cyanidin as much as a 'lossof-function' in terms of delphinidin. This view suggests that PL would have more cyanidin in its petals than ' $\mathrm{CP}$ ', as the mutant $\mathrm{F}^{\prime}{ }^{\prime} 5^{\prime} \mathrm{H}$ is predicted to act as an $\mathrm{F}^{\prime} \mathrm{H}$. This is what is observed (Figure 3b).

\section{Acknowledgements}

We would like to thank David Kaplan for help in the growth and maintenance of Lathyrus odoratus plants. Lina Madilao carried out the LC analysis and Meixuezi Tong assisted with Arabidopsis transformation. We thank Drs Joerg Bohlmann and Xin Li for very kindly facilitating this. We thank Dr Sæmundur Sveinsson for performing the analysis of the transcriptomic data. We gratefully acknowledge the support of an NSERC discovery grant to QC.

\section{References}


Bak, S., Beisson, F., Bishop, G., Hamberger, B., Höfer, R., Paquette, S., and Werck-Reichhart, D. 2011. Cytochromes P450. The Arabidopsis Book: e0144.

Barik, D.P., Mohapatra, U., and Chand, P.K. 2005. Transgenic grasspea (Lathyrus sativus L.): factors influencing Agrobacterium-mediated transformation and regeneration. Plant Cell Reports 24: 523-531.

Barnes, H.J., Arlotto, M.P., and Waterman, M.R. 1991. Expression and enzymatic activity of recombinant cytochrome P450 17 alpha-hydroxylase in Escherichia coli. Proc Natl Acad Sci U S A 88: 5597-601.

Bateson, W., Saunders, E.R., and Punnett, R.C. 1906. Further experiments on inheritance in sweet peas and stocks: Preliminary account. Proc. R. Soc., Lond., Ser. B 77: 236-238.

Beale, G.H., Robinson, G.M., Robinson, R., and Scott-Moncrieff, R. 1939. Genetics and chemistry of flower colour variation in Lathyrus odoratus. Journal of Genetics 37: 375.

Benveniste, I., Lesot, A., Hasenfratz, M.P., Kochs, G., and Durst, F. 1991. Multiple forms of NADPH-cytochrome P450 reductase in higher plants. Biochemical and Biophysical Research Communications 177: 105-112.

Borevitz, J.O., Xia, Y., Blount, J., Dixon, R.A., and Lamb, C. 2000. Activation tagging identifies a conserved MYB regulator of phenylpropanoid biosynthesis. Plant Cell 12: 2383-2394.

Clough, S.J., and Bent, A.F. 1998. Floral dip: a simplified method for Agrobacterium-mediated transformation of Arabidopsis thaliana. Plant J. 16: 735-43.

Falginella, L., Castellarin, S., Testolin, R., Gambetta, G., Morgante, M., and Di Gaspero, G. 2010. Expansion and subfunctionalisation of flavonoid $3^{\prime}, 5^{\prime}$-hydroxylases in the grapevine lineage. BMC Genomics 11: 562. 
Forkmann, G., and Ruhnau, B. 1987. Distinct substrate-specificity of dihydroflavonol 4reductase from flowers of Petunia hybrida. Zeitschrift Fur Naturforschung C - a Journal of Biosciences 42: 1146-1148.

He, H., Ke, H., Keting, H., Qiaoyan, X., and Silan, D. 2013. Flower colour modification of chrysanthemum by suppression of F3'H and overexpression of the exogenous Senecio cruentus F3'5'H gene. PLoS ONE 8: e74395.

Hoekema, A., Kastelein, R.A., Vasser, M., and de Boer, H.A. 1987. Codon replacement in the PGK1 gene of Saccharomyces cerevisiae: experimental approach to study the role of biased codon usage in gene expression. Mol. Cell. Biol., 7: 2914-24.

Johnson, E.T., Ryu, S., Yi, H., Shin, B., Cheong, H., and Choi, G. 2001. Alteration of a single amino acid changes the substrate specificity of dihydroflavonol 4-reductase. Plant J. 25: $325-333$.

Johnson, E.T., Yi, H., Shin, B., Oh, B.-J., Cheong, H., and Choi, G. 1999. Cymbidium hybrida dihydroflavonol 4-reductase does not efficiently reduce dihydrokaempferol to produce orange pelargonidin-type anthocyanins. Plant J. 19: 81-85.

Katsumoto, Y., Fukuchi-Mizutani, M., Fukui, Y., Brugliera, F., Holton, T.A., Karan, M., Nakamura, N., Yonekura-Sakakibara, K., Togami, J., Pigeaire, A., Tao, G.Q., Nehra, N.S., Lu, C.Y., Dyson B.K., Tsuda, S., Ashikari, T., Kusumi, T., Mason, J.G., and Tanaka Y. 2007. Engineering of the rose flavonoid biosynthetic pathway successfully generated blue-hued flowers accumulating delphinidin. Plant and Cell Physiology 48: 1589-1600. 
Lawrence, W.J.C., Price, J.R., Robinson, G.M., and Robinson, R. 1941. The distribution of anthocyanins in flowers, fruits and leaves. Phil. Trans. R. Soc., Lond., Ser. B 230: 149178.

Miller, P. 1754. The gardeners dictionary, volume 2. Ed. 4. London: Privately Printed.

Mizutani, M., and Ohta, D. 1998. Two isoforms of NADPH:cytochrome P450 reductase in Arabidopsis thaliana. Gene structure, heterologous expression in insect cells, and differential regulation. Plant Physiol. 116: 357-67.

Moreau, C., Ambrose, M.J., Turner, L., Hill, L., Ellis, T.H.N., and Hofer, J.M.I. 2012. The b gene of pea (Pisum sativum L.) encodes a defective flavonoid 3' 5' hydroxylase and confers pink flower colour. Plant Physiol. 159: 759-768.

Okinaka, Y., Shimada, Y., Nakano-Shimada, R., Ohbayashi, M., Kiyokawa, S., and Kikuchi, Y. 2003. Selective accumulation of delphinidin derivatives in tobacco using a putative flavonoid 3',5'-hydroxylase cDNA from Campanula medium. Biosci. Biotechnol. Biochem. 67: 161-5.

Plotkin, J.B., and Kudla, G. 2011. Synonymous but not the same: the causes and consequences of codon bias. Nat. Rev. Genet. 12: 32-42.

Punnett, R.C. 1923. Linkage in the sweet pea (Lathyrus odoratus). Journal of Genetics 13: 101124.

Rat'kin, A.V., and Tarasov, V.A. 2010. Genetic control of biosynthesis of anthocyans in sweet pea (Lathyrus odoratus L.) flowers. Russian Journal of Genetics 46: 431-438.

Redouté, P.J. 1833. Choix des plus belles fleurs et des plus beaux fruits. Paris: Ernest Panckoucke, 
Rupasinghe, S., Baudry, J., and Schuler, M.A. 2003. Common active site architecture and binding strategy of four phenylpropanoid P450s from Arabidopsis thaliana as revealed by molecular modeling. Protein Engineering 16: 721-731.

Schoenbohm, C., Martens, S., Eder, C., Forkmann, G., and Weisshaar, B. 2000. Identification of the Arabidopsis thaliana flavonoid 3'-hydroxylase gene and functional expression of the encoded P450 enzyme. Biological Chemistry 381: 749-753.

Seitz, C., Ameres, S., and Forkmann, G. 2007. Identification of the molecular basis for the functional difference between flavonoid 3'-hydroxylase and flavonoid 3',5'-hydroxylase. FEBS Letters 581: 3429-3434.

Seitz, C., Eder, C., Deiml, B., Kellner, S., Martens, S., and Forkmann, G. 2006. Cloning, functional identification and sequence analysis of flavonoid 3'-hydroxylase and flavonoid 3',5'-hydroxylase cDNAs reveals independent evolution of flavonoid 3',5'-hydroxylase in the Asteraceae family. Plant Molecular Biology 61: 365-381.

Shi, M.Z., and Xie, D.Y. 2010. Features of anthocyanin biosynthesis in pap1-D and wild-type Arabidopsis thaliana plants grown in different light intensity and culture media conditions. Planta 231: 1385-400.

Stich, K., Eidenberger, T., Wurst, F., and Forkmann, G. 1992. Enzymatic conversion of dihydroflavonols to flavan-3,4-diols using flower extracts of Dianthus caryophyllus L. (carnation). Planta 187: 103-108.

Švábová, L., and Griga, M. 2008. The effect of cocultivation treatments on transformation efficiency in pea (Pisum sativum L.). Plant Cell, Tissue and Organ Culture 95: 293-304. 
Takeda, K., Osakabe, A., Saito, S., Furuyama, D., Tomita, A., Kojima, Y., Yamadera, M., and Sakuta, M. 2005. Components of protocyanin, a blue pigment from the blue flowers of Centaurea cyanus. Phytochemistry 66: 1607-1613.

Tanaka, Y., and Ohmiya, A. 2008. Seeing is believing: engineering anthocyanin and carotenoid biosynthetic pathways. Curr. Opin. Biotechnol. 19: 190-7.

Tanaka, Y., Fukui, Y., Fukuchimizutani, M., Holton, T.A., Higgins, E., and Kusumi, T. 1995. Molecular cloning and characterization of Rosa hybrida dihydroflavonol 4-reductase gene. Plant And Cell Physiology 36: 1023-1031.

Tohge, T., Nishiyama, Y., Hirai, M.Y., Yano, M., Nakajima, J., Awazuhara, M., Inoue, E., Takahashi, H., Goodenowe, D.B., Kitayama, M., Noji, M., Yamazaki, M., and Saito, K. 2005. Functional genomics by integrated analysis of metabolome and transcriptome of Arabidopsis plants over-expressing an MYB transcription factor. Plant J. 42: 218-35.

Urban, P., Mignotte, C., Kazmaier, M., Delorme, F., and Pompon, D. 1997. Cloning, yeast expression, and characterization of the coupling of two distantly related Arabidopsis thaliana NADPH-cytochrome P450 reductases with P450 CYP73A5. J. Biol. Chem. 272: 19176-86.

Varadarajan, J., Guilleminot, J., Saint-Jore-Dupas, C., Piegu, B., Chaboute, M.E., Gomord, V., Coolbaugh, R.C., Devic, M., and Delorme, V. 2010. ATR3 encodes a diflavin reductase essential for Arabidopsis embryo development. New Phytol. 187: 67-82.

Wessinger, C.A., and Rausher, M.D. 2014. Predictability and irreversibility of genetic changes associated with flower color evolution in Penstemon barbatus. Evolution 68: 1058-70.

Wessinger, C.A., and Rausher, M.D. 2015. Ecological transition predictably associated with gene degeneration. Molecular Biology and Evolution 32: 347-354. 
Winkel-Shirley, B. 2001. Flavonoid biosynthesis. A colorful model for genetics, biochemistry, cell biology, and biotechnology. Plant Physiol. 126: 485-493.

Woollacott, C.M. 2010. The genetic control of petal morphology in the sweet pea (Lathyrus odoratus). MSc thesis, University of British Columbia, Vancouver. 


\section{TABLES}

Table 1. Peak assignment for the analysis of the aqueous methanol extract of the flowers of Lathyrus odoratus, based on the flavonoid aglycone standards. $\mathrm{T}_{\mathrm{R}}$ (retention time) decreases as polarity of the molecule gets higher. More polar molecules elute faster in a reverse phase LC. $\mathrm{m} / \mathrm{z}$ (mass to charge ratio) calculation is based on the mass database at massbank.jp. +/- indicates the mode the compound is detected with. Absorption maxima followed manufacturer's certificate of analysis.

\begin{tabular}{|l|c|c|c|}
\hline Pigment & $\boldsymbol{t}_{\mathbf{R}}(\mathbf{m i n})$ & $\mathbf{m} / \mathbf{z}$ & $\mathbf{U V} \boldsymbol{\lambda}_{\max }(\mathbf{n m})$ \\
\hline Cyanidin & 1.4 & $287(+)$ & 538 \\
\hline Peonidin & 2.8 & $301(+)$ & 537 \\
\hline Delphinidin & 0.9 & $303(+)$ & 548 \\
\hline Malvidin & 3.1 & $331(+)$ & 547 \\
\hline Petunidin & 1.8 & $317(+)$ & 547 \\
\hline Quercetin & 4.5 & $301(-)$ & 372 \\
\hline Kaempferol & 5.8 & $285(-)$ & 365 \\
\hline Myricetin & 2.8 & $317(-)$ & 374 \\
\hline
\end{tabular}


Table 2. F2 co-segregation analyzed with respect to the colour phenotype and F3'5' H genotype in a total of $40 \mathrm{~F} 2$ individuals obtained from the cross ' $\mathrm{CP}$ ' (purple) $\times$ 'PL' (pink). Pink is the recessive phenotype (purple:pink, expected = 3:1) and "a" (the 'PL' F3'5'H marker) is the recessive genotype. " $\mathrm{A}$ ” is the ' $\mathrm{CP}$ ' $\mathrm{F} 3$ '5' $\mathrm{H}$ allele.

\begin{tabular}{|l|l|l|}
\hline F3'5'H genotype & Pink floral phenotype & Purple floral phenotype \\
\hline aa & 11 & 0 \\
\hline Aa & 0 & 22 \\
\hline AA & 0 & 7 \\
\hline Total & 11 & 29 \\
\hline
\end{tabular}




\section{FIGURE LEGENDS}

Figure 1. Top panel: (A) Lathyrus odoratus wild type cultivar 'CP' and (B) pink mutant 'PL'. Bottom panel: transverse section of the standard petal showing an epidermal location of the anthocyanins in 'CP' (C) and 'PL' (D). Magnification $\times 200$.

Figure 2. Intermediates in the flavonoid pathway starting with flavanones and leading to anthocyanidin synthesis. Flavanones (naringenin, eriodyctyol and pentahydroxy flavanone) are converted by flavone 3-hydroxylase (F3H) into dihydroflavonols DHK (dihydrokaempferol), DHQ (dihydroquercetin) and DHM (dihydromyricetin), which can be further converted to either flavonols by flavonol synthase (FLS), or to anthocyanidins, catalysed by dihydroflavonol reductase (DFR) and anthocyanin synthase (ANS). Further methylation of cyanidin and delphinidin into their respective derivatives are carried out by $O$-methyltransferase (OMT). The colour of the end products of each pathway is indicated. The pathway as represented here follows standard references (e.g. Winkel-Shirley 2001; Tanaka and Ohmiya 2008).

Figure 3. (A) The six flower ontogeny stages from small bud (S1) to anthesis (S6). Top panel, $L$. odoratus cv. 'CP' (wild type). Bottom panel, Lathyrus odoratus cv. 'PL' (pink mutant). S1, small bud without visible anthocyanin, bud curving down towards the pedicel. S2, anthocyanin pigment starts to form. S3, anthocyanin is clearly visible. S4, a mature bud forms and it continues to curve away from the pedicel as it enlarges. S5, beginning anthesis, opening of the bud is accompanied by an upright positioning of the flower, now along the same axis as the pedicel. S6, full anthesis, open flower with clear separation of standard and wings. Scale bar represents $1 \mathrm{~cm}$. (B) Pigment profile for ' $\mathrm{CP}$ ' and 'PL', measured at S5-6 stage. Relative abundance is measured as peak area under each identified MS peak.

Figure 4. (A) RT-PCR of $F 3^{\prime} 5^{\prime} H$ expression in wild type 'CP' and pink mutant 'PL' showing no substantial difference. b, standard petal, w, wings. (B) F2 co-segregation analysis digestion gel. The wild type allele is digested into two fragments by restriction enzyme HhaI whereas the mutant allele is not cleaved. 'CP' phenotypes were a mixture of 22 heterozygotes and 7 homozygotes for the $F 3^{\prime} 5^{\prime} H$ ' $\mathrm{CP}$ ' allele (not all ' $\mathrm{CP}$ ' individuals were shown here). 
Figure 5. (A) Chromatogram of $F 3^{\prime} 5^{\prime} H$ showing the missense mutation changing the small uncharged amino acid glycine (in 'CP') into a large and negatively charged aspartic acid (in 'PL'). (B) Multi-species alignment showing the single nucleotide substitution is in a highly conserved position of the SRS1 region across plant species.

Figure 6. Arabidopsis PAPID (a dominant mutant line that produces anthocyanins constitutively) transformed with sweet pea $F 3^{\prime} 5^{\prime} H$ showed elevated levels of cyanidin production, but no delphinidin production. (A) RT-PCR showing the expression of sweet pea $F 3^{\prime} 5^{\prime} H$ was successfully induced in the transgenic lines. (B) Abundance of flavonoid aglycones in leaves and stems from the control plants and the transgenic lines. Col $\times$ papld: control, from crossing Columbia and homozygote $P A P 1-D .35 \mathrm{~s}: \mathrm{cpF} 3^{\prime} 5^{\prime} \mathrm{H}, P A P 1 D$ transformed with wild type $F 3^{\prime} 5^{\prime} H$. 35s:plF3'5'H, PAPID transformed with mutant $F 3^{\prime} 5^{\prime} H$. 

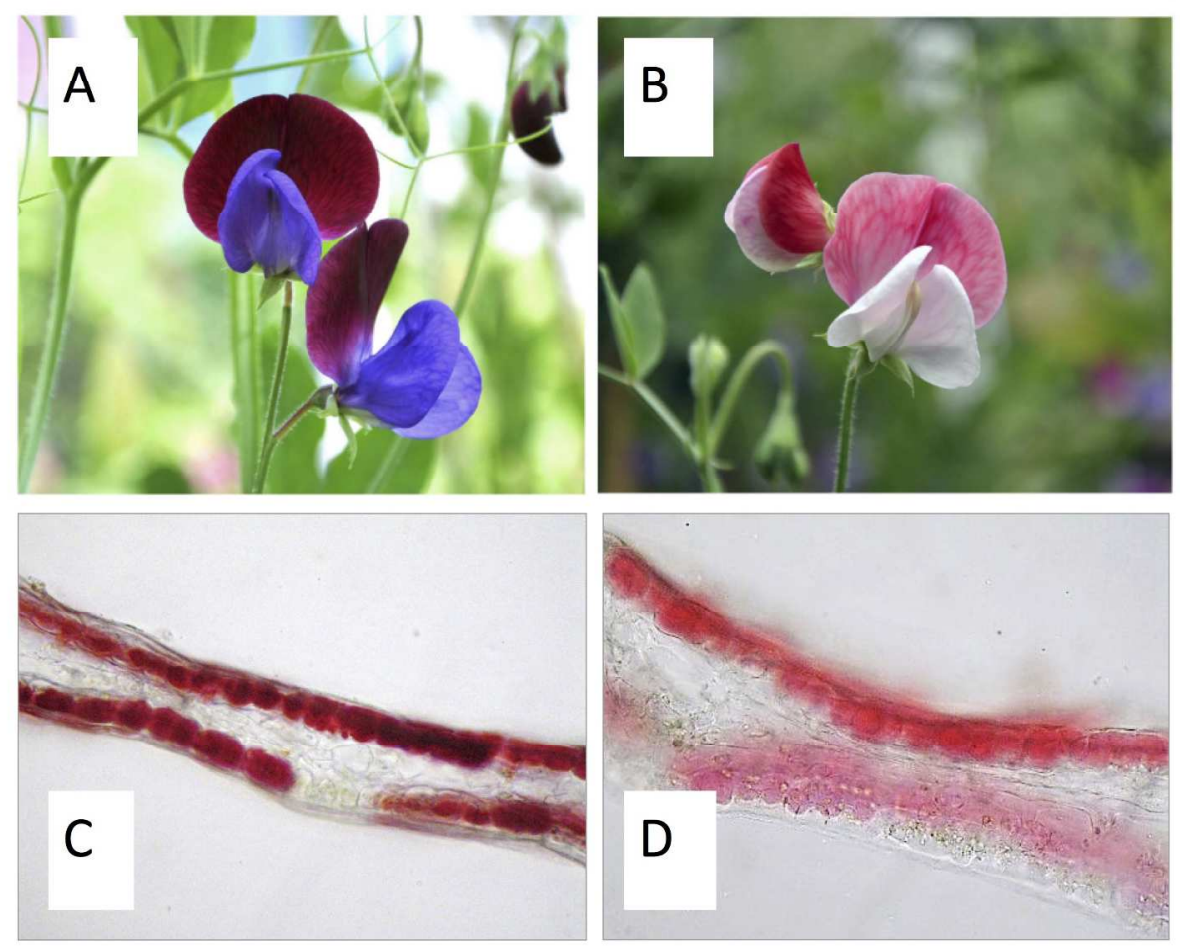

$224 \times 182 \mathrm{~mm}(300 \times 300 \mathrm{DPI})$ 

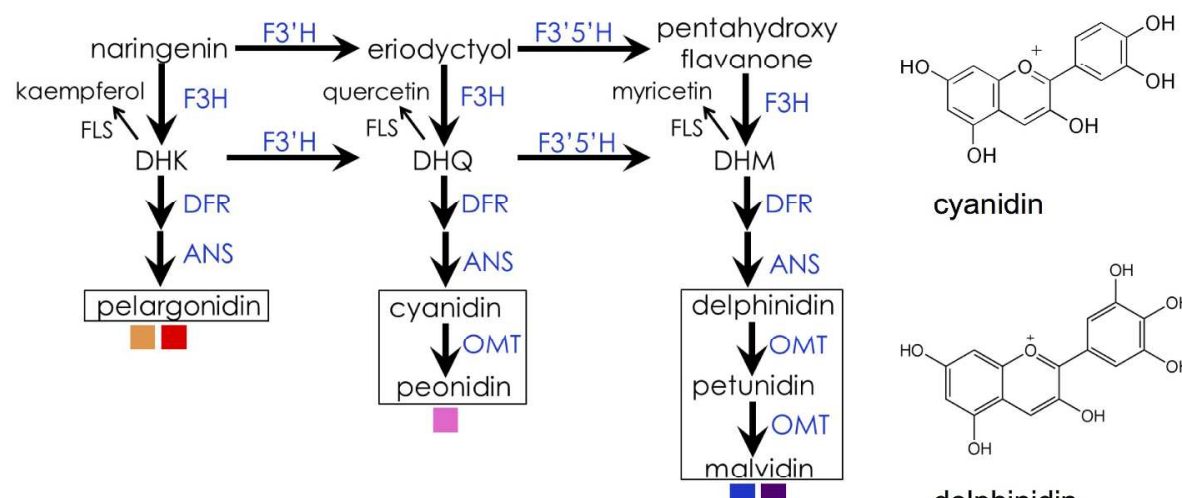

cyanidin

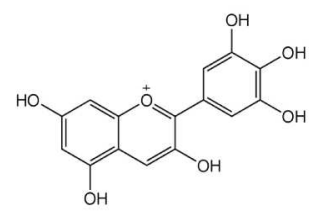

delphinidin

$243 \times 128 \mathrm{~mm}(300 \times 300$ DPI $)$ 

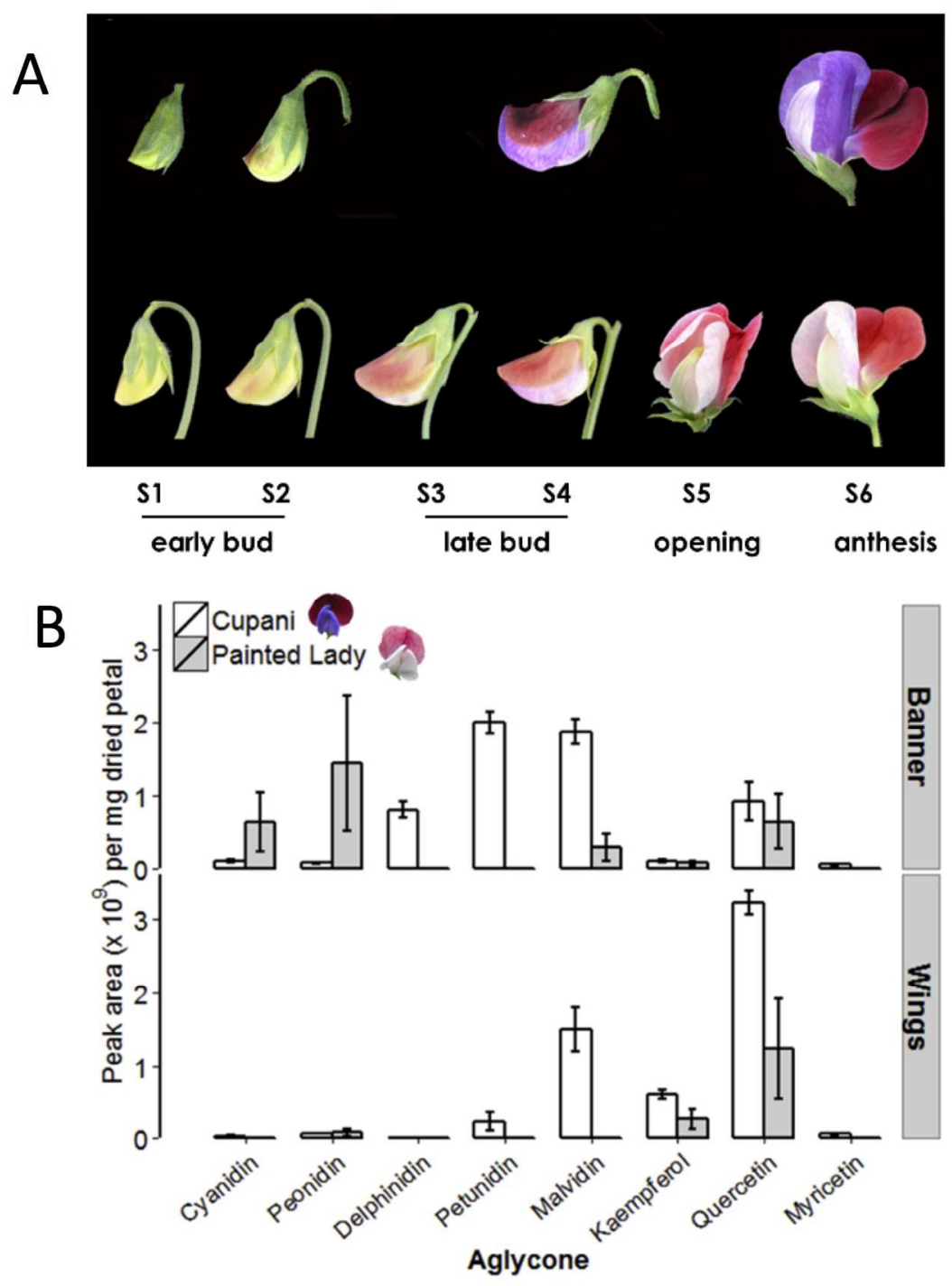

161×199mm (300 x 300 DPI) 


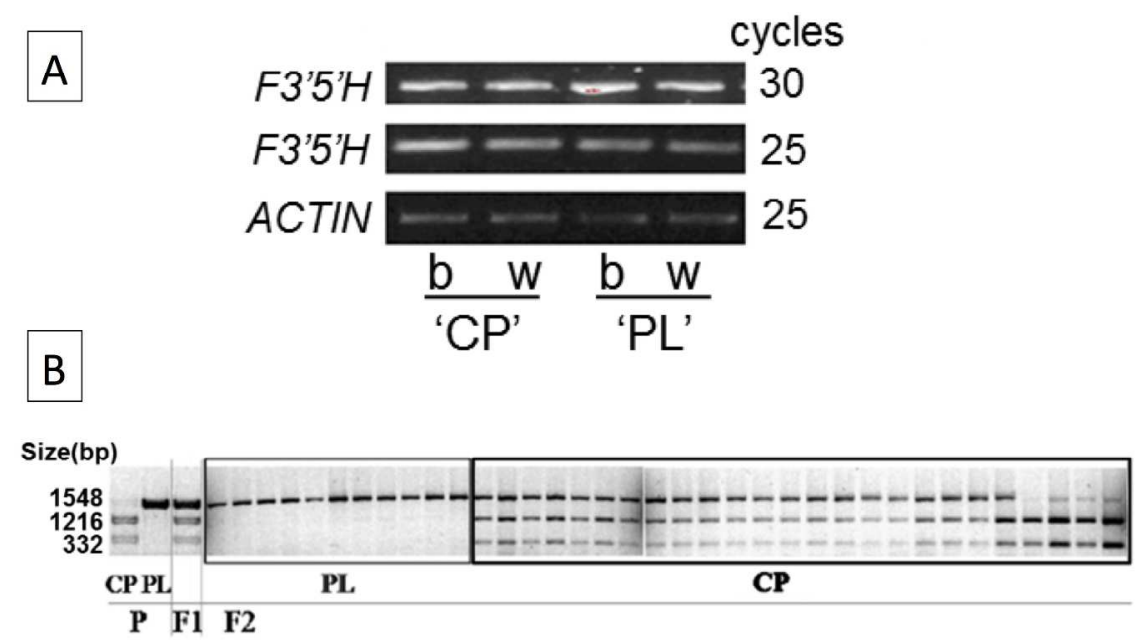

$253 \times 149 \mathrm{~mm}(300 \times 300 \mathrm{DPI})$ 

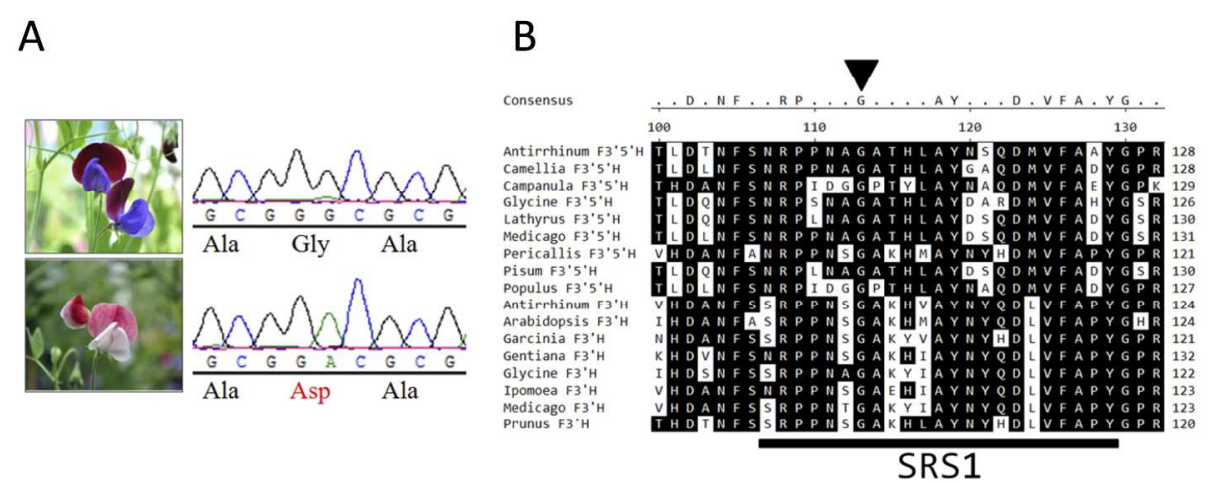

$254 \times 110 \mathrm{~mm}(300 \times 300$ DPI $)$ 
A

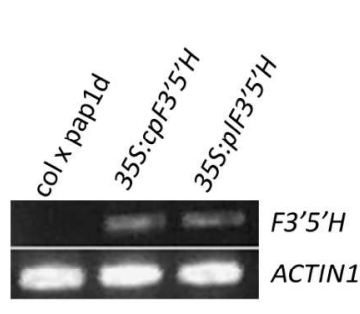

B

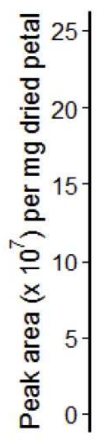
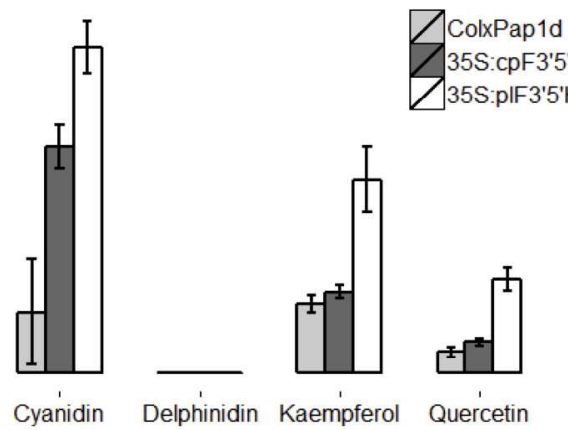
$35 \mathrm{~S}: \mathrm{cpF} 3^{\prime} 5^{\prime} \mathrm{H}$ 35S:plF'5'

Cyanidin

elphinidin Kaempferol Quercetin

$264 \times 131 \mathrm{~mm}(300 \times 300 \mathrm{DPI})$ 